\title{
MAXIMUM LIKELIHOOD IDENTIFICATION OF STOCHASTIC WIENER-HAMMERSTEIN-TYPE NON-LINEAR SYSTEMS
}

\author{
C. H. Chen and S. D. Fassois \\ Department of Mechanical Engineering and Applied Mechanics, The University of Michigan, \\ Ann Arbor, Michigan 48109-2125, U.S.A.
}

(Received 12 November 1990, accepted 28 March 1991)

\begin{abstract}
The identification problem for non-linear Wiener-Hammerstein-type systems is considered. Unlike alternative techniques that are based on deterministic system representations, a stochastic model structure that explicitly accounts for both the input-output and noise dynamics is postulated. The uniqueness properties of this structure are analysed, and appropriate necessary and sufficient conditions derived. A new time-domain identification method based on the Maximum Likelihood principle is then introduced. Unlike alternative approaches that are mainly in the frequency and correlation domains, the proposed method offers statistically optimal estimates from a single record of normal operating data, and is capable of operating directly on the time-domain data and overcoming errors associated with the evaluation of correlation functions/Fourier transforms or multi-stage procedures. The effectiveness and accuracy of the proposed method are verified via numerical simulations with a number of different systems and noise to signal ratios.
\end{abstract}

\section{INTRODUCTION}

Most physical and engineering systems are in reality non-linear, and although they may admit an approximate linear representation in a restricted operating range, they can, in general, be adequately characterised only through appropriate non-linear models. As is well-known, oftentimes this characterisation has to be done via identification techniques that operate on available input-output data.

For the identification of non-linear systems, methods that are based on functional series expansions (such as the Volterra and Wiener series), specific forms of non-linear differential/difference equations, semi-linear models with signal-dependent parameters, blockoriented forms, and other appropriate representations, have been considered Billings [1].

This work is concerned with the identification of block-oriented non-linear systems, and specifically stochastic Wiener-Hammerstein-type systems of the form illustrated in Fig. 1. This form is of particular physical significance as it provides an appropriate representation for systems that are often encountered in engineering practice, and are composed of two linear dynamic elements separated by a static (memory-less) nonlinearity and have their output corrupted by zero-mean stationary random noise characterised by a rational spectral density function. This class of systems also includes the well-known [1] Hammerstein and Wiener representations as special cases.

Although non-linear identification methods based on functional series expansions of the system under study can, in principle, be applied to the identification of WienerHammerstein-type systems, they are by no means preferable as they are faced with problems of excessive computations, very cumbersome model structure selection procedures, and are characterised by significant weaknesses such as their inability to preserve 


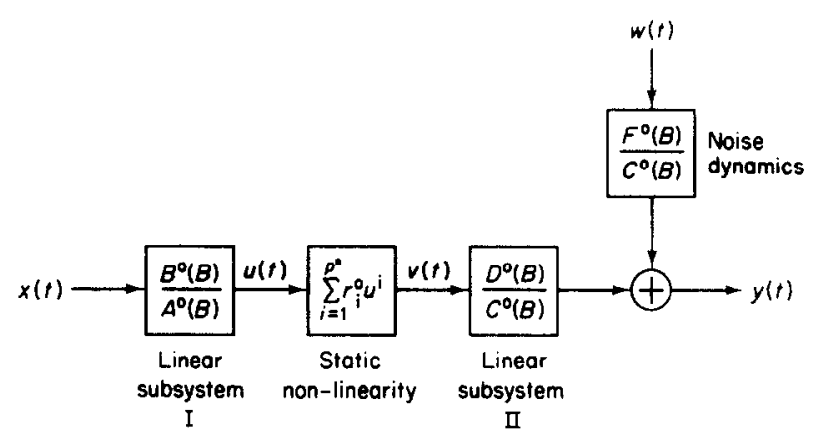

Figure 1. Block diagram representation of the non-linear system $\mathscr{S}$.

the structure of the system under study, incorporate a priori information, and relate estimated quantities to important physical characteristics; all of them due to their black box-type nature. Specialized methods appropriate for the identification of WienerHammerstein-type systems are therefore needed. Such methods can offer significantly reduced computational complexity, and, by preserving the structure of the system to be identified, they can overcome the aforementioned drawbacks of the black-box-type methods and provide a very concise and physically meaningful representation of the underlying physical system.

As a consequence, significant interest has been generated, and a number of such methods have been developed in the past several years. Most of them operate on the correlation or frequency domains, and, either implicitly or explicitly, make use of the fact that the Volterra (or other appropriate) kernels of a Wiener-Hammerstein-type system can be factored, and the factors related to the components of the original system. Also, although some of these methods do account for random noise effects, they are essentially restricted to the deterministic case in the sense that they do not postulate stochastic WienerHammerstein-type representations that explicitly account for both the input-output and noise dynamics and are desirable in many applications involving modeling, analysis, prediction, diagnosis, and control. In addition, as it will be shortly seen, they cannot operate on normal operating data records as they require especially designed excitations, and, in certain cases, multiple tests. A brief account of the various identification approaches that are currently available is given in the sequel. It should be noted that only the approaches that have been developed for Wiener-Hammerstein-type systems are reviewed here; for a review of identification methods applicable to simple Wiener, Hammerstein, or other block-oriented non-linear systems, the reader is referred to the detailed survey papers of Billings [1] and Billings and Fakhouri [2] and the references therein.

A class of identification methods for Wiener-Hammerstein-type systems is based on the work of Lee and Schetzen [3], who derived expressions for the computation of the Wiener kernels of non-linear systems based on input-output cross-correlations. Isobe and Sato [4] have developed a method capable of extracting the magnitude and phase information of the linear transfer functions and the non-linearity characteristics from these kernels. The identification procedure, has however, to be repeated several times, noise effects are not accounted for, a number of errors are typically present in the kernel estimates [5], and estimation of the linear subsystem transfer functions from the magnitude and phase estimates requires the incorporation of additional steps in this approach.

On the other hand, a number of methods [6-9] have been developed based on identification of the linear and higher-order Volterra kernels. These methods, however, often result in excessive experimentation time as they require a number of experiments 
with different types of inputs to be conducted. Korenberg $[10,11]$ has proposed a correlation method for the identification of each one of the system components by using a single experiment under white Gaussian excitation. Billings and Fakhouri [12] have extended earlier results on separable processes and used them to formulate a correlation method that can be used with white Gaussian excitation, and, similarly to Korenberg's method, separates the static non-linearity from the impulse response functions of the linear dynamic subsystems. The linear transfer functions and non-linearity parameters are subsequently estimated by a multi-stage least-squares algorithm. This method has been extended to operate with pseudo-random inputs [13] and Gaussian non-white excitations [14]. A comprehensive summary of these results is provided in Billings and Fakhouri [2]. In other related developments Parker and Moore [15] proposed a modified Volterra series approach based on correlation analysis that allows for the identification of Wiener-Hammerstein-type systems by using three-level input signals of small amplitude and non-zero bias level. De Boer et al. [16] simplified the correlation analysis by using a representation of the static non-linearity in terms of Hermite polynomials, and Korenberg [17] showed that the correlation method under Gaussian excitation will not be affected by the presence of additive random noise in the system input and output variables (the former noise assumed to be propagating through the system).

Some alternative approaches that have been proposed include those of Cooper and Falkner [18], who used time-domain data obtained from a series of experiments with pulse excitations of different amplitudes, and Yasui [19], who used an orthogonal Fourier functional representation of the system.

From this brief overview it is evident that the currently available methods for the identification of Wiener-Hammerstein-type systems are in the correlation or frequency domains, require careful selection of the excitation signals, and, in certain cases, multi-test procedures. As a consequence, a number of errors associated with the evaluation of correlation functions or numerical Fourier transforms are present, and can be often magnified by the multi-stage procedures required for the estimation of the linear transfer functions and the static non-linearity characteristics from the originally obtained kernels. In addition, identification based on normal operating data, that is most desirable or even necessary in practice, remains a prohibitive task, and many of the available methods are restricted to the deterministic (no noise) case, while even those that allow for noise effects in the data to be accounted for, do not postulate the identification of a stochastic system model that can provide an appropriate characterisation for both the input-output and noise dynamics.

The main objective of the present work therefore is the introduction of a new method suitable for the accurate identification of stochastic Wiener-Hammerstein-type systems of the form of Fig. 1, and capable of effectively overcoming the limitations of the currently available approaches. The method developed to achieve this objective is based on the Maximum Likelihood principle [20], and offers the following unique advantages:

(i) The ability to accurately estimate stochastic Wiener-Hammerstein-type systems that expicitly account for both the input-output and noise dynamics.

(ii) The ability to perform identification from a single, even short, record of normal operating data corrupted at any realistic noise to signal (N/S) ratio; a very significant advantage for many practical applications.

(iii) The ability to operate directly on time-domain data and simultaneously estimate all of the system parameters in a statistically optimal sense. Errors related to the evaluation of correlation functions, Fourier transforms, and multi-stage procedures are effectively avoided, while a priori system information may be directly incorporated into the identification procedure. 
The rest of this paper is organised as follows: The problem statement, along with a study of the uniqueness properties of the stochastic Wiener-Hammerstein-type model structure postulated, are presented in section 2 , and the proposed identification method in section 3. The effectiveness and accuracy of the proposed method are examined in section 4 by using numerical simulations with a number of different systems and data corrupted at various $\mathrm{N} / \mathrm{S}$ ratios, and the conclusions of this study are finally summarised in section 5 .

\section{PROBLEM STATEMENT AND THE MODEL STRUCTURE}

In this section the precise form of the underlying physical system to be modeled $\mathscr{S}$ and the corresponding model structure $\mathcal{M}$ are introduced, the identification problem is defined, and the uniqueness properties of $\mathcal{M}$ are investigated.

The system to be estimated is composed of a memoryless polynomial non-linearity imbedded between two linear dynamic elements and has its output corrupted by a stationary stochastic noise process characterised by rational spectral density. A formal mathematical representation of this system may be given as follows $\dagger$ :

$$
\mathscr{S}:\left\{\begin{array}{l}
C^{o}(B) \cdot y(t)=D^{o}(B) \cdot v(t)+F^{o}(B) \cdot w(t) \\
v(t)=\sum_{i=1}^{p^{o}} r_{i}^{o} \cdot u^{i}(t) \\
A^{o}(B) \cdot u(t)=B^{o}(B) \cdot x(t)
\end{array}\right.
$$

where $\{x(t)\},\{y(t)\},\{w(t)\}$ represent the input, output, and innovations signals, respectively, $\{u(t)\},\{v(t)\}$ the input and output of the static non-linearity, respectively, and $A^{o}(B), B^{o}(B), C^{o}(B), D^{o}(B), F^{o}(B)$ polynomials in the backshift operator $B[B x(t)=$ $x(t-1)]$ and of the respective forms:

$$
\begin{gathered}
A^{o}(B) \triangleq 1+a_{1}^{o} B+\cdots+a_{n a^{o}}^{o} B^{n a^{o}} \\
B^{o}(B) \triangleq b_{1}^{o} B+\cdots+b_{n b^{o}}^{o} B^{n b^{o}} \\
C^{o}(B) \triangleq 1+c_{1}^{o} B+\cdots+c_{n c^{o}}^{o} B^{n c^{o}} \\
D^{o}(B) \triangleq d_{1}^{o} B+\cdots+d_{n d^{o}}^{o} B^{n d^{o}} \\
F^{o}(B) \triangleq 1+f_{1}^{o} B+\cdots+f_{n f^{o}}^{o} B^{n f^{o}}
\end{gathered}
$$

The following assumptions are now made:

A1. The polynomials $A^{o}(B)$ and $C^{o}(B)$ are strictly minimum phase (stability conditions), and so is $F^{\circ}(B)$ (invertibility condition).

A2. The innovations signal $\{w(t)\}$ is zero-mean uncorrelated Gaussian, and also uncross-correlated with the excitation $\{x(t)\}$, that is:

$$
E\{w(t)\}=0 \quad E\{w(t) \cdot w(s)\}=\delta_{t, s}\left(\sigma_{w}^{o}\right)^{2} \quad E\{w(t) \cdot x(s)\}=0 \quad \forall t, s
$$

where $E\{\cdot\}$ denotes expectation, $\delta_{t, s}$ the Kronecker delta, and $\left(\sigma_{w}^{o}\right)^{2}$ the variance of $\{w(t)\}$.

A3. The excitation signal $\{x(t)\}$ is persistently exciting of sufficiently high order.

The one-step-ahead minimum-mean-square-error prediction $\hat{y}(t / t-1)$ of $y(t)$ given input and noise-corrupted output data up to time $t-1$, may be then shown to be:

$$
\hat{y}(t / t-1)=\left[1-\frac{C^{o}(B)}{F^{o}(B)}\right] y(t)+\frac{D^{o}(B)}{F^{o}(B)} v(t)
$$

† The superscript $o$ is used to indicate quantities associated with the true system $\mathscr{S}$ and distinguish them from those of candidate models. 
with $v(t)$ given by (1b), (1c), and the corresponding prediction error is:

$$
e(t / t-1) \triangleq y(t)-\hat{y}(t / t-1)=w(t) .
$$

These equations, combined with (1b) and (1c), define the prediction error form of the system.

The problem of concern in this work may be then stated as follows: "Given input and output observations $x_{1}^{N} \underline{\underline{\Delta}}\{x(1), \ldots, x(N)\}$ and $y_{1}^{N} \triangleq\{y(1), \ldots, y(N)\}$, respectively, select a model $\mu(\theta)$ from an appropriate model structure $\mu$, that best matches the input-output and noise characteristics of the actual system $\mathscr{Y}$."

The model structure $\mathcal{M}$ is hereby selected as the set of all models $\mathcal{M}(\theta)$ of the form (compare with the prediction error form of the system):

$$
\mu(\theta):\left\{\begin{array}{l}
e(t / \theta)=y(t)-\hat{y}(t / \theta) \quad \operatorname{Var}[e(t / \theta)]=\sigma_{w}^{2}(\theta) \\
\hat{y}(t / \theta)=\left[1-\frac{C(B, \theta)}{F(B, \theta)}\right] y(t)+\frac{D(B, \theta)}{F(B, \theta)} v(t / \theta) \\
v(t / \theta)=\sum_{i=1}^{p} r_{i}(\theta) \cdot u^{i}(t / \theta) \\
u(t / \theta)=\frac{B(B, \theta)}{A(B, \theta)} x(t)
\end{array}\right.
$$

in which $\theta$ represents the parameter vector to be estimated:

$$
\boldsymbol{\theta} \triangleq\left[\operatorname{coef} A, \operatorname{coef} B, r_{1}, \ldots, r_{p}, \operatorname{coef} C, \operatorname{coef} D, \operatorname{coef} F, \sigma_{w}^{2}\right]^{T},
$$

$\hat{y}(t / \theta)$ the one-step-ahead prediction based on the model, and $e(t / \theta)$ the corresponding prediction error. The polynomials $A(B, \theta), B(B, \theta), C(B, \theta), D(B, \theta)$, and $F(B, \theta)$ are assumed to be of degrees $n a, n b, n c, n d$, and $n f$, respectively, and of the respective forms indicated in expression (2). The model structure may be then formally defined as:

$$
\mathscr{M} \triangleq\left\{\mathscr{M}(\boldsymbol{\theta}) / \boldsymbol{\theta} \in \mathscr{H}(A) \times \mathscr{R}^{n b} \times \mathscr{R}^{p} \times \mathscr{H}(C) \times \mathscr{R}^{n d} \times \mathscr{H}(F) \times \mathscr{R}^{+}\right\}
$$

in which $\mathscr{H}(A), \mathscr{H}(C)$, and $\mathscr{H}(F)$ represent those subspaces of $\mathscr{R}^{n a}, \mathscr{R}^{n c}$, and $\mathscr{R}^{n f}$ for which the polynomials $A(B, \theta), C(B, \theta)$, and $F(B, \theta)$, respectively, are strictly minimum phase.

Before proceeding with the development of the identification method, it is necessary to investigate the conditions under which the system $S$ can be accurately and uniquely represented within the model structure $\mu$. This is important as it is clearly desirable to ensure both the existence and uniqueness $\dagger$ of a model $\boldsymbol{M ( \theta )} \in \mathcal{M}$ such that $\boldsymbol{\mu ( \theta )}=\mathscr{S}$.

In order to derive necessary and sufficient conditions for both existence and uniqueness, the system and model representations are rewritten as:

$$
\begin{aligned}
\mathscr{S}: & y(t)=f^{o}\left(x^{t-2}, w^{t}\right) \\
\mathcal{M}(\boldsymbol{\theta}): & y(t)=f\left(x^{t-2}, e^{t}, \boldsymbol{\theta}\right)
\end{aligned}
$$

where

$$
\begin{aligned}
& x^{t-2} \triangleq\{x(t-2), x(t-3), \ldots\}, \\
& w^{t} \triangleq\{w(t), w(t-1), \ldots\}, \\
& e^{t} \triangleq\{e(t / \theta), e(t-1 / \theta), \ldots\},
\end{aligned}
$$

$\dagger$ It is well known that in case of non-uniqueness severe numerical problems may occur during the implementation of any appropriate estimation procedure. 
and $\mathscr{D}(\mathscr{Y}, \mathcal{M})$ is defined as the set of those parameter vectors $\theta$ for which $\mathcal{M}(\theta)=\mathscr{Y}$, that is:

$$
\mathscr{D}(\mathscr{S}, \mathcal{M}) \stackrel{\Delta}{\underline{\Delta}}\left\{\boldsymbol{\theta} / f^{o}\left(x^{t-2}, w^{t}\right)=f\left(x^{t-2}, e^{t}, \boldsymbol{\theta}\right),\left(\sigma_{w}^{o}\right)^{2}=\sigma_{w}^{2}\right\}
$$

The problem is then equivalent to that of determining the necessary and sufficient conditions for $\mathscr{D}(\mathscr{Y}, \mathcal{M})$ to be empty or consist of one or more elements.

In achieving this objective the following additional conditions are imposed on $\mathscr{Y}$ :

A4. The polynomials $A^{o}(B), B^{\circ}(B)$, as well as $C^{\circ}(B), D^{o}(B), F^{o}(B)$ have no common factors.

A5. The leading coefficients of the $B^{\circ}(B)$ and $D^{\circ}(B)$ polynomials are equal to unity $\left(b_{1}^{o}=d_{1}^{o}=1\right)$.

The first condition is required in order to ensure the controllability and observability of the two linear subsystems and the noise transfer function, whereas the second condition is necessary for achieving uniqueness. This may be readily seen by compactly rewriting the system equations (1) as:

$$
\begin{aligned}
\mathscr{S}: y(t)= & \left(b_{1}^{o} d_{1}^{o} r_{1}^{o}\right) \cdot\left[C^{o}(B)\right]^{-1} \cdot \overline{D^{o}}(B) \cdot \sum_{i=1}^{p^{o}} \overline{r_{i}^{o}} \cdot\left(b_{1}^{o}\right)^{i-1}\left[\left[A^{o}(B)\right]^{-1} \cdot \overline{B^{o}}(B) \cdot x(t)\right]^{i} \\
& +\left[C^{o}(B)\right]^{-1} \cdot F^{o}(B) \cdot w(t)
\end{aligned}
$$

where

$$
\overline{B^{o}}(B) \stackrel{\Delta}{\underline{\Delta}}\left(b_{1}^{o}\right)^{-1} \cdot B^{o}(B), \quad \overline{D^{o}}(B) \stackrel{\Delta}{\underline{y}}\left(d_{1}^{o}\right)^{-1} \cdot D^{o}(B),
$$

and

$$
\overline{r_{i}^{o}} \stackrel{\Delta}{=}\left(r_{1}^{o}\right)^{-1} \cdot r_{i}^{o}(i=1,2, \ldots, p) .
$$

From this expression it is evident that the static gain of the system is proportional to the product $b_{1}^{o} d_{1}^{o} r_{1}^{o}$. In order to achieve a unique parameterisation, and without any loss of generality, the parameters $b_{1}^{o}$ and $d_{1}^{o}$ are thus set equal to unity.

The problems of existence and uniqueness are then dealt with in the following proposition:

Proposition: Existence and Uniqueness

Consider the system $\mathscr{S}$ of equations (1) subject to assumptions (A1)-(A5), the model structure $\mathcal{M}$ of equation (8), and the set $\mathscr{D}(\mathscr{S}, \mathcal{M})$ defined by equation (11). Also let $n_{1}^{*} \stackrel{\Delta}{\min }\left\{n a-n a^{o}, n b-n b^{o}\right\} \quad$ and $n_{2}^{*} \triangleq \min \left\{n c-n c^{o}, n d-n d^{o}, n f-n f^{o}\right\}$. The set $\mathscr{D}(\mathscr{S}, \mathcal{M})$ then consists of:

a. no elements if and only if $\min \left\{p-p^{o}, n_{1}^{*}, n_{2}^{*}\right\}<0$

b. exactly one element if and only if $p-p^{\circ} \geqslant 0$ and $n_{1}^{*}=n_{2}^{*}=0$

c. infinitely many elements if and only if $\min \left\{p-p^{o}, n_{1}^{*}, n_{2}^{*}\right\} \geqslant 0$ and $\max \left\{n_{1}^{*}, n_{2}^{*}\right\}>0$.

Proof: By using (A1) the system equations (1) may be compactly written as:

$$
\begin{aligned}
\mathscr{S}: \quad y(t)= & {\left[C^{o}(B)\right]^{-1} \cdot D^{o}(B) \cdot\left[\sum_{i=1}^{p^{o}} r_{i}^{o}\left\{\left[A^{o}(B)\right]^{-1} \cdot B^{o}(B) \cdot x(t)\right\}^{i}\right] } \\
& +\left[C^{o}(B)\right]^{-1} \cdot F^{o}(B) \cdot w(t) .
\end{aligned}
$$

By defining the impulse response functions:

$$
\begin{aligned}
& H_{1}^{o}(B) \stackrel{\Delta}{=}\left[A^{o}(B)\right]^{1} \cdot B^{o}(B)=B+h_{12}^{o} B^{2}+\cdots \\
& H_{2}^{o}(B) \triangleq\left[C^{o}(B)\right]^{-1} \cdot D^{o}(B)=B+h_{22}^{o} B^{2}+\cdots \\
& H_{3}^{o}(B) \triangleq\left[C^{o}(B)\right]^{-1} \cdot F^{o}(B)=1+h_{31}^{0} B+h_{32}^{o} B^{2}+\cdots
\end{aligned}
$$


in which (A5) was already accounted for, (13) may be alternatively expressed as:

$$
\text { S: } \begin{aligned}
y(t)= & f^{o}\left(x^{t-2}, w^{t}\right)=\sum_{i=1}^{p^{o}} r_{i}^{o} \sum_{k=1}^{\infty} h_{2 k}^{o} \sum_{l_{1}=1}^{\infty} \cdots \sum_{l i=1}^{\infty} h_{1 l_{1}}^{o} \cdot h_{1 l_{2}}^{o} \cdots h_{1 l_{i}}^{o} . \\
& x\left(t-l_{1}-k\right) \cdot x\left(t-l_{2}-k\right) \cdots x\left(t-l_{i}-k\right)+\sum_{k=0}^{\infty} h_{3 k}^{o} \cdot w(t-k) .
\end{aligned}
$$

The model form $\mathscr{M}(\theta)$ can be analogously expressed, and the identity $\mathscr{S}=\mathscr{M}(\theta)$ then becomes:

$$
\begin{aligned}
\sum_{i=1}^{p^{o}} r_{i}^{o} & \sum_{k=1}^{\infty} h_{2 k}^{o} \sum_{l_{1}=1}^{\infty} \cdots \sum_{l_{i}=1}^{\infty} h_{1 l_{1}}^{o} \cdot h_{1 l_{2}}^{o} \cdots h_{1 l_{t}}^{o} \cdot x\left(t-l_{1}-k\right) \cdot x\left(t-l_{2}-k\right) \cdots x\left(t-l_{i}-k\right) \\
& +\sum_{k=0}^{\infty} h_{3 k}^{o} \cdot w(t-k) \\
= & \sum_{i=1}^{p} r_{i}(\theta) \sum_{k=1}^{\infty} h_{2 k}(\theta) \sum_{l_{1}=1}^{\infty} \cdots \sum_{l_{i}=1}^{\infty} h_{1 l_{1}}(\theta) \cdot h_{1 l_{2}}(\theta) \cdots h_{1 l_{t}}(\theta) \cdot x\left(t-l_{1}-k\right) \cdot x\left(t-l_{2}-k\right) \\
& \cdots x\left(t-l_{i}-k\right)+\sum_{k=0}^{\infty} h_{3 k}(\theta) \cdot e(t-k / \theta) .
\end{aligned}
$$

By assuming, for the moment, that the impulse responses $\left\{h_{1 k}(\theta)\right\},\left\{h_{2 k}(\theta)\right\},\left\{h_{3 k}(\theta)\right\}$ can be freely determined and that $p-p^{\circ} \geqslant 0$, it is obvious that equation (16) will have at least one solution (the true one) in terms of the non-linearity coefficients and the aforementioned impulse responses. It will be now shown that this solution is unique. Towards this end it is sufficient to consider only a subset of the identities implied by equation (16).

Indeed, by considering the coefficients of the terms $x^{i}(t-2)$ for $i=1,2, \ldots, p$ in both sides of equation (16), one obtains:

$$
r_{i}(\theta)=r_{i}^{o} \quad(i=1,2, \ldots, p) .
$$

For the impulse responses $\left\{h_{1 k}\right\}$ and $\left\{h_{2 k}\right\}$ any one of the non-linear terms (quadratic, cubic, etc.) can be considered. By considering, for instance, the coefficients of the terms $x(t-2) \cdot x(t-i)$ for $i=3,4, \ldots$ in both sides of equation (16), the expressions:

$$
r_{2}(\theta) \cdot h_{1(i-1)}(\theta)=r_{2}^{o} \cdot h_{1(i-1)}^{o}
$$

are obtained, from which:

$$
h_{1 i}(\theta)=h_{1 i}^{o} \quad(i=1,2, \ldots) .
$$

By considering the coefficients of the terms $x^{2}(t-i)$ for $i=3,4, \ldots$, the expressions:

$$
r_{2}(\theta) \cdot \sum_{k=1}^{i-1} h_{2 k}(\theta) \cdot h_{1(i-k)}^{2}(\theta)=r_{2}^{o} \cdot \sum_{k=1}^{i-1} h_{2 k}^{o} \cdot\left(h_{1(i-k)}^{o}\right)^{2}
$$

are obtained, which, given equations (17a) and (17b), yield:

$$
h_{2 i}(\theta)=h_{2 i}^{o} \quad(i=1,2, \ldots) .
$$

Finally, by considering the coefficients of the white noise terms, one obtains:

$$
h_{3 i}(\theta)=h_{3 i}^{o} \quad(i=1,2, \ldots) .
$$

Since the subset of equations considered admits only one solution, namely the true one given by expressions (17), and the totality of equations implied by equation (16) has at least one solution, it is readily concluded that expressions (17) indeed compose the unique solution of equation (16). 
The problem therefore becomes equivalent to finding the conditions, in terms of $p, p^{\circ}$, and the system and model polynomials, under which the totality of equations (17) may be simultaneously met for one or more values of the parameter vector $\boldsymbol{\theta}$. Towards this objective, and by using the definitions (14), the conditions (17) may be equivalently expressed as:

$$
\begin{gathered}
r_{i}(\boldsymbol{\theta})=r_{i}^{o} \quad(i=1,2, \ldots, p) \\
A(B, \boldsymbol{\theta}) \cdot B^{o}(B)-A^{o}(B) \cdot B(B, \theta)=0 \\
C(B, \theta) \cdot D^{o}(B)-C^{o}(B) \cdot D(B, \theta)=0 \\
C(B, \boldsymbol{\theta}) \cdot F^{o}(B)-C^{o}(B) \cdot F(B, \theta)=0
\end{gathered}
$$

a. Existence: A parameter vector $\theta$ simultaneously satisfying equations (18) will exist if and only if:

$$
\begin{gathered}
p-p^{o} \geqslant 0 \\
n_{1}^{*}=\min \left\{n a-n a^{o}, n b-n b^{o}\right\} \geqslant 0 \\
n_{2}^{*}=\min \left\{n c-n c^{o}, n d-n d^{o}, n f-n f^{o}\right\} \geqslant 0 .
\end{gathered}
$$

The first condition is required for equation (18a), the second for (18b), and the third for the combination of $(18 \mathrm{c})$ and $(18 \mathrm{~d})$.

b. Uniqueness: By using assumption (A4) it may be shown that a unique parameter vector $\theta$ simultaneously satisfying all of equations (18) will exist if and only if:

$$
\begin{gathered}
p-p^{\circ} \geqslant 0 \\
n_{1}^{*}=\min \left\{n a-n a^{o}, n b-n b^{\circ}\right\}=0 \\
n_{1}^{*}=\min \left\{n c-n c^{o}, n d-n d^{\circ}, n f-n f^{o}\right\}=0
\end{gathered}
$$

The first of these conditions is required in order for the model to allow for at least as many non-linear terms as the actual system, whereas the second and third conditions may be shown to guarantee the existence of unique polynomials $A(B, \theta)$ and $B(B, \theta)$ satisfying equation (18b), and $C(B, \theta), D(B, \theta), F(B, \theta)$ satisfying equations (18c)-(18d), respectively.

c. Multiple solutions: Multiple solutions will be obtained if and only if $p-p^{\circ} \geqslant 0$ (required for existence) and there exist more than one set of polynomials satisfying equations (18b) and/or (18c)-(18d). Based on assumption (A4), it may be shown that equation (18b) will have infinitely many solutions of the form:

$$
A(B, \theta)=A^{o}(B) \cdot L(B, \theta) \quad B(B, \theta)=B^{o}(B) \cdot L(B, \theta)
$$

with $L(B, \theta)$ being an arbitrary monic polynomial of degree $n_{1}^{*}=\min \left\{n a-n a^{o}, n b-n b^{o}\right\}$, if and only if $n_{1}^{*}>0$. Similarly, equations (18c)-(18d) will have infinitely many solutions of the form:

$$
\begin{aligned}
& C(\boldsymbol{B}, \boldsymbol{\theta})=C^{o}(\boldsymbol{B}) \cdot L(\boldsymbol{B}, \boldsymbol{\theta}) \\
& D(\boldsymbol{B}, \boldsymbol{\theta})=D^{o}(\boldsymbol{B}) \cdot L(\boldsymbol{B}, \boldsymbol{\theta}) \\
& F(\boldsymbol{B}, \boldsymbol{\theta})=F^{o}(\boldsymbol{B}) \cdot L(\boldsymbol{B}, \boldsymbol{\theta})
\end{aligned}
$$

with $L(B, \theta)$ being again an arbitrary monic polynomial of degree $n_{2}^{*}=$ $\min \left\{n c-n c^{o}, n d-n d^{o}, n f-n f^{o}\right\}$, if and only if $n_{2}^{*}>0$. By also considering the existence conditions (19) it may be concluded that infinitely many solutions will exist if and only if:

$$
p-p^{o} \geqslant 0 \quad \min \left\{n_{1}^{*}, n_{2}^{*}\right\} \geqslant 0 \quad \max \left\{n_{1}^{*}, n_{2}^{*}\right\}>0 .
$$




\section{THE IDENTIFICATION METHOD}

The problem of estimating a model $M(\theta)$ of the form (6) from available input and noise-corrupted output observations $x_{1}^{N}$ and $y_{1}^{N}$, respectively, is now considered. The proposed method is based on the Maximum Likelihood (ML) principle [20], as it is well-known that ML-based estimation approaches are characterised by optimal asymptotic $(N \rightarrow \infty)$ properties (namely consistency and efficiency) under rather mild conditions [20].

Towards this objective the conditional likelihood function is constructed by assuming that the innovations sequence $\{w(t)\}$ is Gaussian. Based on the prediction error form of the system and model, the conditional probability density of $e(t / \theta)$ given all the data up to time $t-1$ may be shown to be also Gaussian and of the form:

$$
f_{e(t) /(t-1)}(e(t / \theta) / t-1)=\frac{1}{\sqrt{2 \pi \sigma_{w}^{2}}} \exp \left[-\frac{1}{2 \sigma_{w}^{2}}[e(t / \theta)]^{2}\right] \quad \forall t \in \mathscr{Z}^{+} .
$$

The conditional likelihood function may be then expressed as:

$$
L\left(x_{1}^{N}, y_{1}^{N}, \theta\right)=\prod_{t=1}^{N} f_{e(t) /(t-1)}(e(t / \theta) / t-1)
$$

which, by using equation (6a) and standard procedures [20], may be shown to be optimised for:

$$
\begin{gathered}
\hat{\theta}_{1}=\arg \min V_{N}\left(\theta_{1}\right) \triangleq \arg \min \frac{1}{N} \sum_{t=1}^{N} \frac{1}{2} e^{2}\left(t / \theta_{1}\right) \\
\hat{\sigma}_{w}^{2}=\frac{1}{N} \sum_{t=1}^{N} e^{2}\left(t / \hat{\theta}_{1}\right)
\end{gathered}
$$

In these expressions $\theta_{1}$ is defined by the partition:

$$
\theta \triangleq\left[\theta_{1}^{T}: \sigma_{w}^{2}\right]^{T}
$$

and arg min denotes minimising argument.

Because of the non-quadratic nature of the dependence of $V_{N}\left(\theta_{1}\right)$ on $\theta_{1}$, the estimator (23a) cannot be put into a closed-form; instead $V_{N}\left(\theta_{1}\right)$ has to be numerically optimised. A general iterative approach that may be used for such optimization problems is of the form [21]:

$$
\hat{\boldsymbol{\theta}}_{1}^{(i+1)}=\hat{\boldsymbol{\theta}}_{1}^{(i)}-\mu^{(i)} \cdot\left[\mathbf{R}^{(i)}\right]^{-1} \cdot \mathbf{V}_{N}^{\prime}\left(\hat{\boldsymbol{\theta}}_{1}^{(i)}\right)
$$

where $\hat{\boldsymbol{\theta}}_{1}^{(i)}$ denotes. the estimate obtained in the $i$-th iteration, $\mu^{(i)}$ the step size selected such that $V_{N}\left(\hat{\boldsymbol{\theta}}_{1}^{(i+1)}\right)<V_{N}\left(\hat{\boldsymbol{\theta}}_{1}^{(i)}\right), \mathbf{R}^{(i)}$ a square matrix of appropriate dimensions that modifies the search direction, and $V_{N}^{\prime}\left(\theta_{1}\right)$ the gradient of $V_{N}\left(\theta_{1}\right)$ given as:

$$
V_{N}^{\prime}\left(\theta_{1}\right) \triangleq \frac{d V_{N}\left(\theta_{1}\right)}{d \theta_{1}} \triangleq-\frac{1}{N} \sum_{t=1}^{N} \psi\left(t / \theta_{1}\right) \cdot e\left(t / \theta_{1}\right)
$$

with:

$$
\psi\left(t / \theta_{1}\right) \triangleq \frac{\mathrm{d} \hat{y}\left(t / \theta_{1}\right)}{\mathrm{d} \theta_{1}}=-\frac{\mathrm{d} e\left(t / \theta_{1}\right)}{\mathrm{d} \theta_{1}}
$$

For the computation of the negative gradient of the one-step-ahead prediction error with respect to $\theta_{1}$, the following expression is obtained by combining equations (6a) and (6b):

$$
-F\left(B, \theta_{1}\right) \cdot e\left(t / \theta_{1}\right)=D\left(B, \theta_{1}\right) \cdot v\left(t / \theta_{1}\right)-C\left(B, \theta_{1}\right) \cdot y(t)
$$


which may be subsequently differentiated with respect to all the elements of $\theta_{1}$. Indeed, differentiation with respect to $a_{i}$ yields:

$$
-F(B) \frac{\partial e(t)}{\partial a_{i}}=\sum_{k=1}^{n d} \mathrm{~d}_{k} \frac{\partial v(t-k)}{\partial a_{i}}
$$

where the argument $\boldsymbol{\theta}_{1}$ has been dropped for simplicity of presentation. By using the fact that the non-linearity is static and differentiable [equation (6c)], (29) may be rewritten as:

$$
\begin{aligned}
-F(B) \frac{\partial e(t)}{\partial a_{i}} & =\sum_{k=1}^{n d} \mathrm{~d}_{k} \frac{\partial v(t-k)}{\partial u(t-k)} \frac{\partial u(t-k)}{\partial a_{i}} \\
& =\sum_{k=1}^{n d} \sum_{m=1}^{p} \mathrm{~d}_{k} \cdot m \cdot r_{\dot{m}}[u(t-k)]^{m-1} \cdot q_{i}(t-k) \quad(i=1, \ldots, n a)
\end{aligned}
$$

where

$$
q_{i}(t-k) \triangleq \frac{\partial u(t-k)}{\partial a_{i}} \quad(i=1, \ldots, n a) .
$$

The derivatives $q_{i}(t-k)$ may be computed by differentiating (6d) to obtain:

$$
\begin{gathered}
u(t-k-i)+A(B) \frac{\partial u(t-k)}{\partial a_{i}}=0 \\
\Leftrightarrow \quad A(B) \cdot q_{i}(t-k)=-u(t-k-i) \quad(i=1, \ldots, n a)
\end{gathered}
$$

which may be solved recursively in time for the sequence $\left\{q_{i}(t)\right\}$.

Next, by differentiating (28) with respect to $b_{i}$ and using (6c), the following expression is obtained:

$$
\begin{aligned}
-F(B) \frac{\partial e(t)}{\partial b_{i}} & =\sum_{k=1}^{n d} \mathrm{~d}_{k} \frac{\partial v(t-k)}{\partial b_{i}}=\sum_{k=1}^{n d} \mathrm{~d}_{k} \frac{\partial v(t-k)}{\partial u(t-k)} \frac{\partial u(t-k)}{\partial b_{i}} \\
& =\sum_{k=1}^{n d} \sum_{m=1}^{p} d_{k} \cdot m \cdot r_{m} \cdot[u(t-k)]^{m-1} \cdot s_{i}(t-k) \quad(i=1, \ldots, n b)
\end{aligned}
$$

where

$$
s_{i}(t-k) \triangleq \frac{\partial u(t-k)}{\partial b_{i}} \quad(i=1, \ldots, n b) .
$$

The derivative $s_{i}(t-k)$ may be computed by using equation (6d) as follows:

$$
\begin{gathered}
A(B) \cdot \frac{\partial u(t-k)}{\partial b_{i}}=x(t-k-i) \\
\Leftrightarrow \quad A(B) \cdot s_{i}(t-k)=x(t-k-i) \quad(i=1, \ldots, n b) .
\end{gathered}
$$

The remaining partial derivatives may be computed from $(28)$ by considering the model (6). Indeed:

$$
\begin{gathered}
-F(B) \cdot \frac{\partial e(t)}{\partial r_{i}}=\sum_{k=1}^{n d} d_{k} \frac{\partial v(t-k)}{\partial r_{i}}=\sum_{k=1}^{n d} d_{k} \cdot[u(t-k)]^{i} \quad(i=1, \ldots, p) \\
-F(B) \cdot \frac{\partial e(t)}{\partial c_{i}}=-y(t-i) \quad(i=1, \ldots, n c) \\
-F(B) \cdot \frac{\partial e(t)}{\partial d_{i}}=v(t-i) \quad(i=1, \ldots, n d) \\
\frac{\partial}{\partial f_{i}}[F(B) \cdot e(t)]=0 \Rightarrow-F(B) \cdot \frac{\partial e(t)}{\partial f_{i}}=e(t-i) \quad(i=1, \ldots, n f) .
\end{gathered}
$$


Based on expressions (30), (33), and (36)-(39), the negative gradient of the one-stepahead prediction error with respect to $\theta_{1}$ may be expressed as:

$$
F(B) \cdot \psi\left(t / \theta_{1}\right)=\phi\left(t / \theta_{1}\right)
$$

where the vector $\phi\left(t / \theta_{1}\right)$ is defined as:

$$
\boldsymbol{\phi}\left(t / \boldsymbol{\theta}_{1}\right)=\left[\begin{array}{c}
\sum_{k=1}^{n d} \sum_{m=1}^{p} d_{k} \cdot m \cdot r_{m} \cdot[u(t-k)]^{m-1} \cdot q_{1}(t-k) \\
\vdots \\
\sum_{k=1}^{n d} \sum_{m=1}^{p} d_{k} \cdot m \cdot r_{m} \cdot[u(t-k)]^{m-1} \cdot q_{n a}(t-k) \\
\sum_{k=1}^{n d} \sum_{m=1}^{p} d_{k} \cdot m \cdot r_{m} \cdot[u(t-k)]^{m-1} \cdot s_{1}(t-k) \\
\vdots \\
\sum_{k=1}^{n d} \sum_{m=1}^{p} d_{k} \cdot m \cdot r_{m} \cdot[u(t-k)]^{m-1} \cdot s_{n b}(t-k) \\
\sum_{k=1}^{n d} d_{k} \cdot u(t-k) \\
\vdots \\
\sum_{k=1}^{n d} d_{k} \cdot[u(t-k)]^{p} \\
-y(t-1) \\
\vdots \\
-y(t-n c) \\
v(t-1) \\
\vdots \\
v(t-n d) \\
e(t-1) \\
\vdots \\
e(t-n f) \\
\vdots
\end{array}\right]
$$

with $q_{i}(t)(i=1, \ldots, n a)$ and $s_{i}(t)(i=1, \ldots, n b)$ given by equations (32) and (35), respectively.

Within the context of the proposed method the estimation of the vector $\theta_{1}$ is accomplished by minimising the ML criterion $V_{N}\left(\theta_{1}\right)$ by using a two-phase combined gradient and Gauss-Newton scheme. During the first phase a gradient technique based on equation (25) with:

$$
\mathbf{R}^{(i)}=\mathbf{I}
$$

is used, until the norm of the difference of the parameter vector $\theta_{1}$ in two successive iterations becomes smaller than a prespecified threshold value:

$$
\left\|\hat{\boldsymbol{\theta}}_{1}^{(i)}-\hat{\boldsymbol{\theta}}_{1}^{(i+1)}\right\|<\varepsilon .
$$

The estimate thus obtained is subsequently used as the initial estimate in the second phase which employs a Gauss-Newton technique in which $\mathbf{R}^{(i)}$ is approximated as:

$$
\mathbf{R}^{(i)}=V_{N}^{\prime \prime}\left(\theta_{1}\right) \cong \frac{1}{N} \sum_{t=1}^{N} \psi\left(t / \hat{\theta}_{1}^{(i)}\right) \cdot \psi^{T}\left(t / \hat{\theta}_{1}^{(t)}\right) .
$$


In this expression $V_{N}^{\prime \prime}\left(\theta_{1}\right)$ represents the Hessian of $V_{N}$ with respect to $\theta_{1}$, and the indicated approximation is satisfactory for values of $\hat{\theta}_{1}$ that are 'close' to the actual $\theta_{1}^{\circ}$. For computational purposes this phase is further divided into a number of stages within which different step sizes $\mu^{(i)}$ are used, and is terminated as soon as a convergence criterion of the form (43), with an appropriately selected threshold value, is satisfied.

The proposed method may be now formally summarised as follows:

1. Assume zero initial conditions and assign an appropriately selected initial value to $\boldsymbol{\theta}_{1}$.

2. Select an appropriate step size $\mu^{(i)}$ and set $\mathbf{R}^{(i)}=\mathbf{I}$.

3. Compute $u(t / \theta)$ from equation (6d), $v(t / \theta)$ from (6c), and $e(t / \theta)$ from (6a) and (6b), for $t=1, \ldots, N$.

4. Compute $q_{i}(t) \quad(i=1, \ldots, n a)$ and $s_{i}(t)(i=1, \ldots, n b)$ for $t=1, \ldots, N$ from equations (32) and (35), respectively.

5. Compute $\phi\left(t / \theta_{1}\right)$ for $t=1, \ldots, N$ by using equation (41).

6. Compute $\psi\left(t / \theta_{1}\right)$ for $t=1, \ldots, N$ by using equation (40).

7. Obtain $\hat{\boldsymbol{\theta}}_{1}^{(i+1)}$ by using (25) and (26).

8. Increase the iteration index by one and repeat steps 3-8 until the convergence criterion (43) is satisfied.

9. Select an appropriate step size $\mu^{(i)}$ and compute $\mathbf{R}^{(i)}$ from equation (44).

10. Increase the iteration index by one and perform the computations of steps 3-8.

11. Repeat steps 9 and 10 until a convergence criterion of the form (43) is satisfied.

12. Obtain $\hat{\sigma}_{w}^{2}$ from equation (23b).

Remarks: (a) The two-phase combined gradient and Gauss-Newton scheme of the proposed method has been designed in order to best use the advantages of each technique and circumvent their respective limitations. Hence optimisation is started by using the gradient technique which is computationally simpler, and is later switched to the GaussNewton as the former becomes very slow in the neighbourhood of the optimum.

(b) The optimisation scheme cannot, of course, distinguish between local and global minima. If a local minimum of $V_{N}\left(\theta_{1}\right)$ is reached, the estimated model will be inaccurate, and should be detected as such by the validation procedure outlined in the next section. In that case the estimation will have to be repeated by using a new set of initial guess parameter values.

(c) It should be noted that partial a priori information regarding the system to be identified may be directly accounted for by incorporating it into the model (6) and appropriately modifying the parameter vector $\theta$ to be estimated.

\section{SIMULATION RESULTS AND DISCUSSION}

In this section the performance characteristics of the proposed method are evaluated via numerical simulations. In all cases considered the process response was generated by using mutually independent normal pseudo-random sequences with zero mean and approximately flat spectra acting as input and innovations processes, and the ratio of the variance of the innovations over that of the excitation is defined as the $N / S$ ratio, that is:

$$
\mathrm{N} / \mathrm{S}=\frac{\operatorname{Var}[w(t)]}{\operatorname{Var}[x(t)]} \times 100 \% .
$$

Unless otherwise indicated, in each case data records consisting of 2100 points each were generated, with the first 2000 points composing the estimation and the last 100 the validation subsets. The latter is exclusively used for model validation, which consists of two phases: In the first phase the model-based one-step-ahead predictions of the system 
output, computed via equations (6), are compared to the actual output, whereas in the second phase the uncorrelatedness of the model-based one-step-ahead prediction errors is examined. For a good model these errors must be uncorrelated, and therefore model quality is judged by examining the normalised sample autocorrelation function of the prediction error sequence via a standard statistical test [22].

It is finally noted that in the simulation results that follow, estimation accuracy is judged in terms of parametric error indices, which, for the case of a parameter vector $\theta$, are defined as:

$$
E_{\theta}=\frac{\left\|\hat{\boldsymbol{\theta}}-\theta^{\circ}\right\|_{I I}}{\left\|\theta^{\circ}\right\|_{I I}} \times 100 \%
$$

with $\|\cdot\|_{I}$ indicating octahedric vector norm.

\section{IDENTIFICATION RESULTS}

Table 1 presents identification results for System A with block diagram representation shown in Fig. 2. Three cases, corresponding to $\mathrm{N} / \mathrm{S}$ ratios of 1,10 , and $30 \%$, are considered, and the estimation accuracy is excellent in all of them with errors not exceeding $3.5 \%$. A Monte Carlo analysis of the method with the same system based on 20 data records is presented in Table 2 for the $\mathrm{N} / \mathrm{S}=1 \%$ case, and, as it may be immediately observed, the sample means of the parameter estimates are very close to their actual values (the parametric errors being smaller than $1 \%$ ) and their sample standard deviations quite small.

Table 3 presents estimation results from data records of various lengths $(N=500,1000$, 2000 ) for a somewhat more complicated system (System B) with block diagram representation shown in Fig. 3. Similarly to the previous case three $N / S$ ratios $(1,10$ and $30 \%)$ are considered, and excellent accuracy is achieved in all cases while the method performs equally well with all the data records (the maximum parametric errors in this case barely

TABLE 1

Estimation results for System A

\begin{tabular}{lcccc}
\hline & & \multicolumn{3}{c}{ Estimated parameters } \\
\cline { 3 - 5 } & System & $\mathrm{N} / \mathrm{S}=1 \%$ & $\mathrm{~N} / \mathrm{S}=10 \%$ & $\mathrm{~N} / \mathrm{S}=30 \%$ \\
\hline$a_{1}$ & -0.500 & -0.500 & -0.501 & -0.501 \\
$r_{1}$ & 0.100 & 0.100 & 0.102 & 0.103 \\
$r_{2}$ & -0.700 & -0.700 & -0.700 & -0.698 \\
$c_{1}$ & -0.200 & -0.198 & -0.195 & -0.193 \\
& $E_{A}(\%)$ & 0.00 & 0.20 & 0.20 \\
& $E_{R}(\%)$ & 0.00 & 0.25 & 0.63 \\
& $E_{C}(\%)$ & 1.00 & 2.50 & 3.50 \\
\hline
\end{tabular}

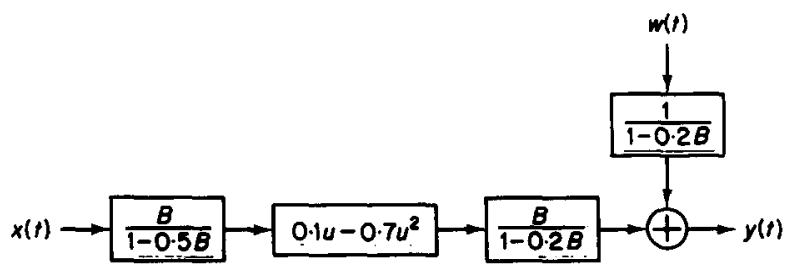

Figure 2. Block diagram representation of System $\mathbf{A}$. 
TABLE 2

Monte Carlo results for System A

\begin{tabular}{|c|c|c|c|}
\hline & \multirow{2}{*}{$\begin{array}{c}\text { System } \\
\text { parameters }\end{array}$} & \multicolumn{2}{|c|}{ Estimated parameters } \\
\hline & & Mean value & Standard deviation \\
\hline$a_{1}$ & -0.500 & -0.501 & 0.0024 \\
\hline$r_{1}$ & $0 \cdot 100$ & 0.103 & 0.0053 \\
\hline$r_{2}$ & -0.700 & -0.700 & 0.0038 \\
\hline$c_{1}$ & $-0 \cdot 200$ & $-0 \cdot 199$ & 0.0050 \\
\hline
\end{tabular}

Number of data sets used: 20

N/S ratio: $1 \%$

Parametric errors: $E_{A}=0.20 \% \quad E_{R}=0.38 \% \quad E_{C}=0.50 \%$

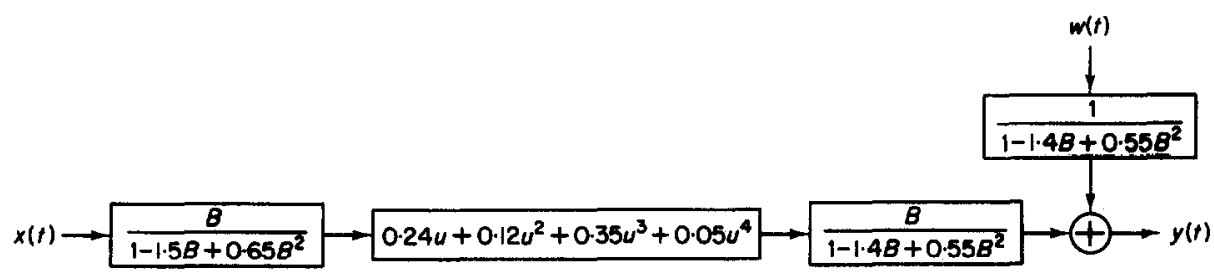

Figure 3. Block diagram representation of System B.
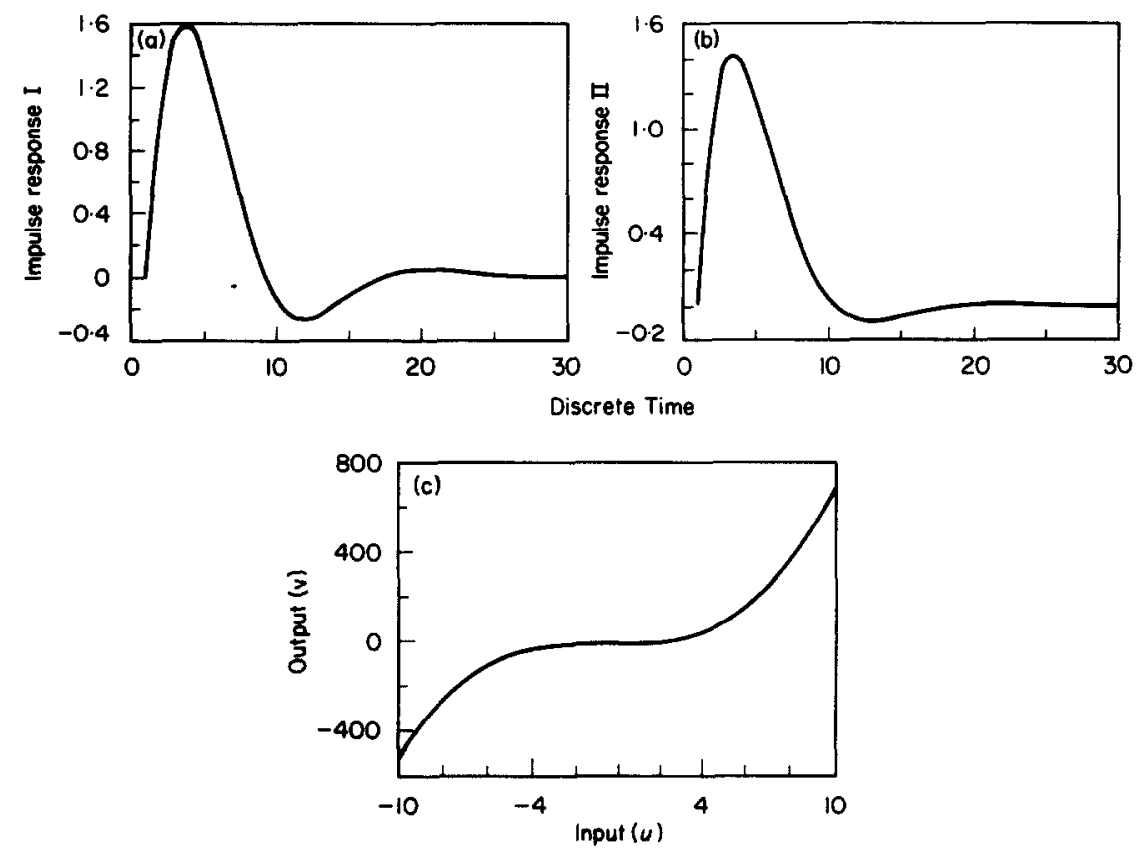

Figure 4. Estimation results at three different $N / S$ ratios (System $B ; N=2000$ ). (a) Impulse response I; (b) impulse response II; (c) static non-linearity. (-: Theoretical; ---: estimated at $N / S=1 \% ; \cdots \cdots:$ estimated at $\mathrm{N} / \mathrm{S}=10 \%$; $-\cdot--$ : estimated at $\mathrm{N} / \mathrm{S}=30 \%$ ). 
TABLE 3

Estimation results for System B with data records of various lengths

\begin{tabular}{|c|c|c|c|c|c|}
\hline \multirow{2}{*}{$\begin{array}{l}\text { Data record } \\
\text { length }\end{array}$} & & \multirow{2}{*}{$\begin{array}{c}\text { System } \\
\text { parameters }\end{array}$} & \multicolumn{3}{|c|}{ Estimated parameters } \\
\hline & & & $N / S=1 \%$ & $N / S=10 \%$ & $\mathrm{~N} / \mathrm{S}=\mathbf{3 0} \%$ \\
\hline \multirow[t]{11}{*}{$N=500$} & $a_{1}$ & $-1 \cdot 500$ & $-1 \cdot 500$ & $-1 \cdot 500$ & $-1 \cdot 500$ \\
\hline & $a_{2}$ & 0.650 & 0.650 & 0.650 & 0.650 \\
\hline & $r_{1}$ & $0 \cdot 240$ & $0 \cdot 240$ & 0.239 & 0.238 \\
\hline & $r_{2}$ & $0 \cdot 120$ & $0 \cdot 120$ & $0 \cdot 121$ & $0 \cdot 121$ \\
\hline & $r_{3}$ & $0 \cdot 350$ & 0.350 & 0.350 & 0.351 \\
\hline & $r_{4}$ & 0.050 & 0.050 & 0.050 & 0.050 \\
\hline & $\overrightarrow{c_{1}}$ & $-1 \cdot 400$ & $-1 \cdot 400$ & -1.400 & $-1 \cdot 401$ \\
\hline & $c_{2}$ & 0.550 & 0.550 & $0 \cdot 550$ & 0.551 \\
\hline & & $E_{A}(\%)$ & 0.00 & 0.00 & 0.00 \\
\hline & & $E_{R}(\%)$ & 0.00 & 0.26 & 0.52 \\
\hline & & $E_{C}(\%)$ & 0.00 & 0.00 & 0.05 \\
\hline \multirow[t]{11}{*}{$N=1000$} & $a_{1}$ & -1.500 & $-1 \cdot 500$ & $-1 \cdot 500$ & -1.500 \\
\hline & $a_{2}$ & $0 \cdot 650$ & 0.650 & 0.650 & 0.650 \\
\hline & $r_{1}$ & $0 \cdot 240$ & $0 \cdot 240$ & 0.239 & 0.238 \\
\hline & $r_{2}$ & $0 \cdot 120$ & $0 \cdot 120$ & $0 \cdot 121$ & $0 \cdot 123$ \\
\hline & $r_{3}$ & 0.350 & $0 \cdot 350$ & 0.350 & 0.350 \\
\hline & $r_{4}$ & 0.050 & 0.050 & 0.050 & 0.050 \\
\hline & $c_{1}$ & $-1 \cdot 400$ & $-1 \cdot 400$ & $-1 \cdot 400$ & -1.400 \\
\hline & $c_{2}$ & 0.550 & $0 \cdot 550$ & 0.550 & 0.551 \\
\hline & & $E_{A}(\%)$ & 0.00 & 0.00 & 0.00 \\
\hline & & $E_{R}(\%)$ & 0.00 & 0.26 & 0.65 \\
\hline & & $E_{C}(\%)$ & 0.00 & 0.00 & 0.05 \\
\hline \multirow[t]{11}{*}{$N=2000$} & $a_{1}$ & $-1 \cdot 500$ & $-1 \cdot 500$ & $-1 \cdot 500$ & $-1 \cdot 500$ \\
\hline & $a_{2}$ & 0.650 & 0.650 & 0.650 & $0 \cdot 650$ \\
\hline & $r_{1}$ & 0.240 & 0.241 & 0.243 & 0.245 \\
\hline & $r_{2}$ & $0 \cdot 120$ & 0.121 & $0 \cdot 122$ & 0.123 \\
\hline & $r_{3}$ & $0 \cdot 350$ & 0.350 & $0 \cdot 350$ & 0.350 \\
\hline & $r_{4}$ & 0.050 & 0.050 & 0.050 & 0.050 \\
\hline & $c_{1}$ & $-1 \cdot 400$ & $-1 \cdot 400$ & $-1 \cdot 400$ & $-1 \cdot 400$ \\
\hline & $c_{2}$ & 0.550 & 0.550 & 0.550 & 0.550 \\
\hline & & $E_{A}(\%)$ & 0.00 & 0.00 & 0.00 \\
\hline & & $E_{R}(\%)$ & 0.26 & 0.66 & 1.05 \\
\hline & & $E_{C}(\%)$ & 0.00 & 0.00 & 0.00 \\
\hline
\end{tabular}

exceed $1 \%$ ). The estimated impulse response functions of the two linear subsystems and the static non-linearity for the $N=2000$ case are depicted in Fig. 4, and are virtually indistinguishable from the theoretical curves for all the N/S ratio cases. Figure 5 presents the model validation results for the $10 \% \mathrm{~N} / \mathrm{S}$ ratio and $N=2000$ case. As it may be readily observed the model-based one-step-ahead predictions are very close to the actual process output, and the normalised sample autocorrelation function of the prediction errors lies within the $\pm 1 \cdot 96 / \sqrt{N}$ band, as required for an accurate model.

Table 4 finally presents estimation results for System $\mathrm{C}$ with block diagram representation shown in Fig. 6. The same three N/S ratios $(1,10$ and $30 \%)$ are considered, and excellent accuracy is once again achieved with parametric errors not exceeding $\mathbf{1} \cdot 5 \%$. 

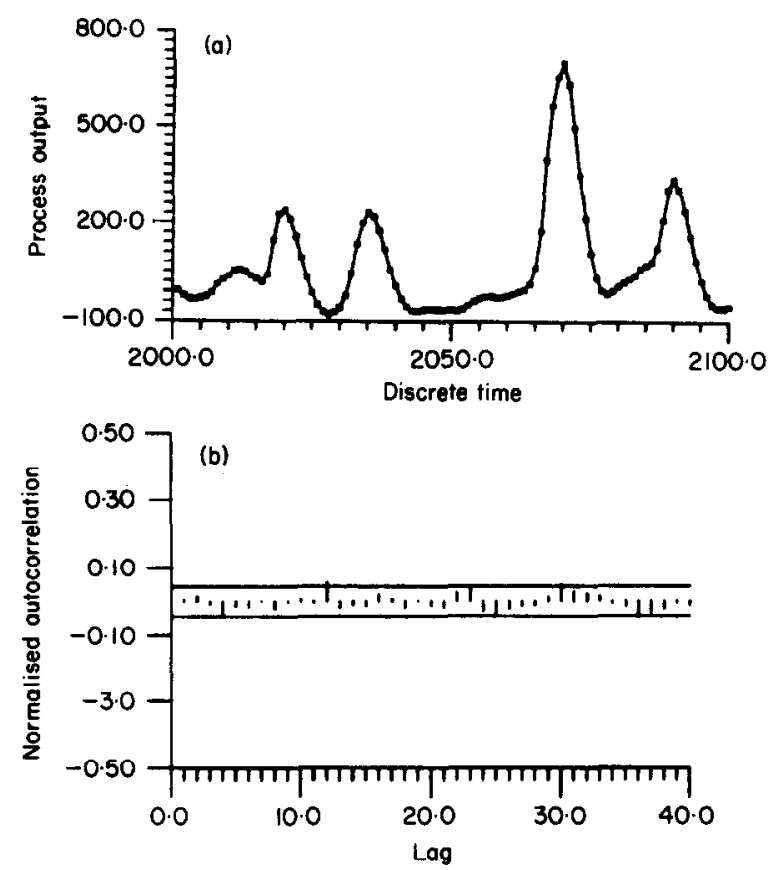

Figure 5. Validation of the estimated model. (a) Model-based one-step-ahead predictions of the system output; (b) the normalised autocorrelation of the residuals. (System B; N/S =10\%;N=2000). - , Process output; , model-based prediction.

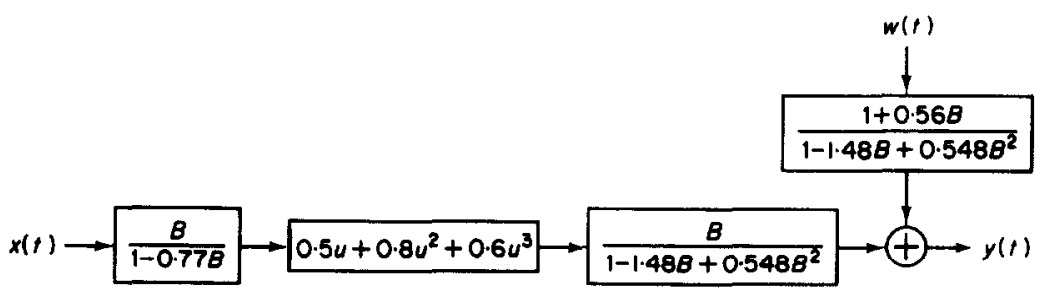

Figure 6. Block diagram representation of System C.

TABLE 4

Estimation results for System C

\begin{tabular}{|c|c|c|c|c|}
\hline & \multirow{2}{*}{$\begin{array}{c}\text { System } \\
\text { parameters }\end{array}$} & \multicolumn{3}{|c|}{ Estimated parameters } \\
\hline & & $N / S=1 \%$ & $\mathrm{~N} / \mathrm{S}=10 \%$ & $\mathrm{~N} / \mathrm{S}=\mathbf{3 0} \%$ \\
\hline$a_{1}$ & -0.770 & -0.770 & -0.771 & -0.771 \\
\hline$r_{1}$ & $0 \cdot 500$ & 0.497 & 0.491 & 0.485 \\
\hline$r_{2}$ & 0.800 & 0.801 & 0.803 & 0.806 \\
\hline$r_{3}$ & 0.600 & 0.600 & 0.599 & 0.598 \\
\hline$c_{1}$ & -1.480 & -1.480 & -1.481 & -1.481 \\
\hline \multirow{6}{*}{$\begin{array}{l}c_{2} \\
f_{1}\end{array}$} & 0.548 & 0.548 & 0.549 & 0.549 \\
\hline & 0.560 & 0.564 & 0.564 & 0.564 \\
\hline & $E_{A}(\%)$ & 0.00 & 0.13 & $0 \cdot 13$ \\
\hline & $E_{R}(\%)$ & $0 \cdot 21$ & 0.68 & 1.21 \\
\hline & $E_{c}(\%)$ & 0.00 & 0.10 & 0.10 \\
\hline & $E_{F}(\%)$ & 0.71 & 0.71 & 0.71 \\
\hline
\end{tabular}



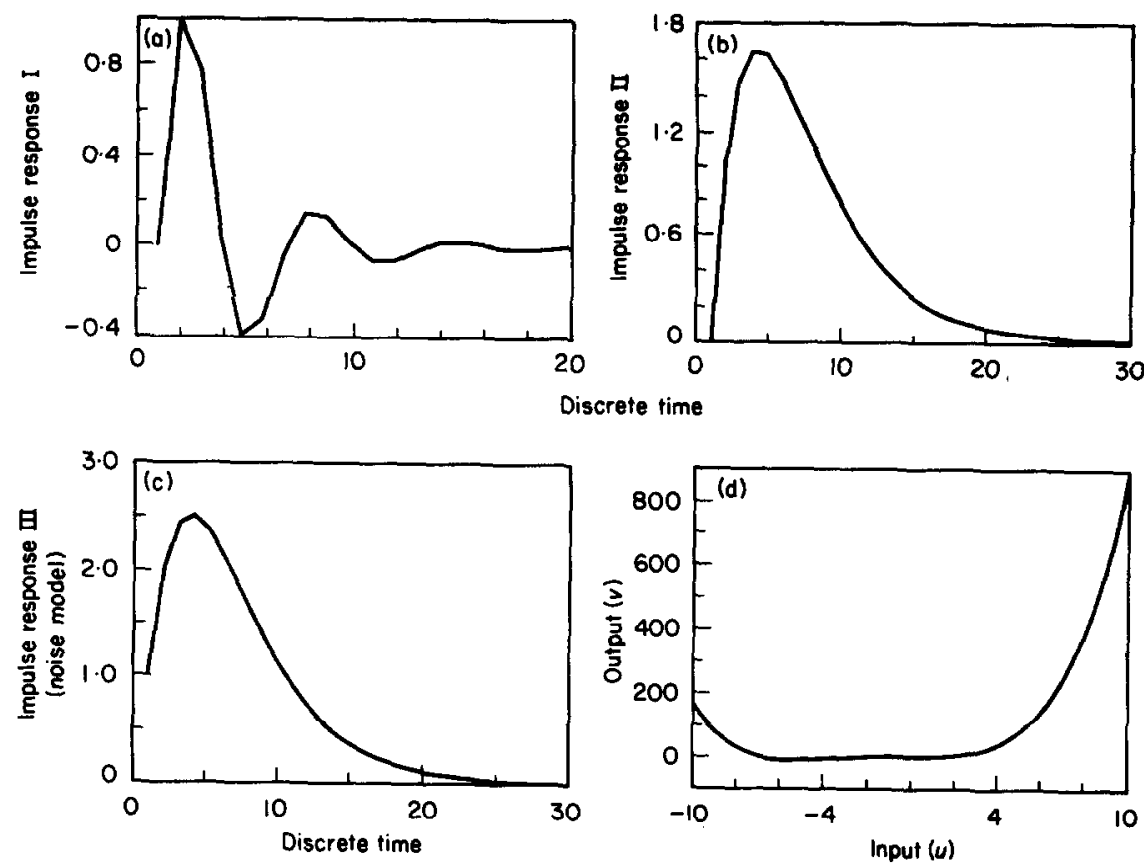

Figure 7. Estimation results at three different $N / S$ ratios (System $C ; N=2000$ ). (a) Impulse response I; (b) impulse response II; (c) impulse response III; (d) static non-linearity. (-: Theoretical; - -: estimated at $\mathrm{N} / \mathrm{S}=1 \% ; \cdots \cdot$ : estimated at $\mathrm{N} / \mathrm{S}=10 \% ;-\cdots-\cdots$ : estimated at $\mathrm{N} / \mathrm{S}=30 \%$ ).
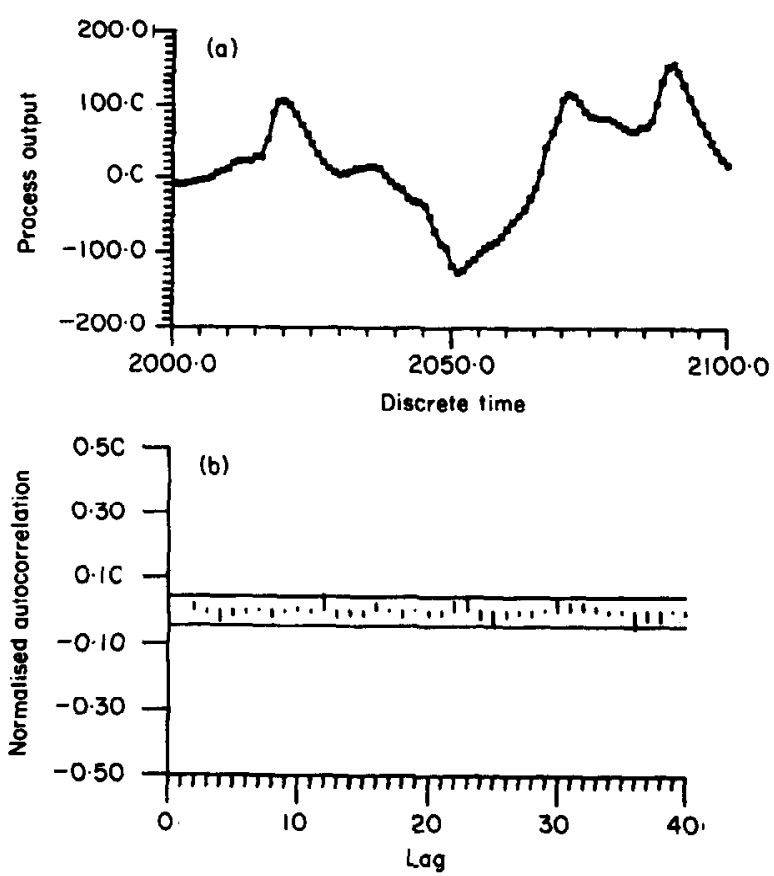

Figure 8. Validation of the estimated model. (a) Model-based one-step-ahead predictions of the system output; (b) the normalised autocorrelation of the residuals. (System $C ; N / S=10 \% ; N=2000$ ). - , Process output; , model-based prediction. 
The estimated impulse response functions of the two linear subsystems and the noise dynamics, as well as the static non-linearity characteristics, are all shown in Fig. 7, and are completely indistinguishable from the theoretical curves for all the $\mathrm{N} / \mathrm{S}$ ratio cases. Figure 8 presents the model validation results for the $10 \% \mathrm{~N} / \mathrm{S}$ ratio case. The model-based one-step-ahead predictions are excellent, and so is the normalised sample autocorrelation function of the corresponding prediction errors.

\section{CONCLUSIONS}

In this paper the identification problem for non-linear Hammerstein-Wiener-type systems was considered based on normal operating data. Unlike alternative methods based on deterministic representations, a stochastic model structure that explicitly accounts for both the input-output and noise dynamics was postulated. This structure offers obvious advantages, and is to be preferred in many applications involving modeling, analysis, prediction, diagnosis and control.

The uniqueness properties of the postulated model structure were analysed, and necessary and sufficient conditions established. The stochastic identification problem was subsequently considered, and a new time-domain method based on the ML principle introduced. This proposed method overcomes the limitations of the currently available approaches that are primarily based on the frequency or correlation domains, by offering:

1. Accurate (consistent and efficient) estimation of stochastic Wiener-Hammersteintype systems.

2. The ability to perform identification based on a single record of normal operating data. No multiple tests or especially designed excitations that are required by alternative approaches are needed.

3. The ability to operate directly on time-domain data and simultaneously estimate all of the system parameters. Errors associated with the evaluation of correlation functions or Fourier transforms and multi-stage procedures for system parameter estimation, as required by some alternative schemes, are completely avoided.

4. Excellent performance characteristics even at high $\mathrm{N} / \mathrm{S}$ ratios and with short data records, and the ability to incorporate available a priori information.

The effectiveness and accuracy of the proposed method were finally demonstrated through numerical simulations with a number of different systems and $N / S$ ratios.

\section{REFERENCES}

1. S. A. Billings 1980 IEE Proceedings 127(D), 272-285. Identification of non-linear systems-A survey.

2. S. A. Billings and S. Y. FAKhouri 1982 Automatica 18, 15-26. Identification of systems containing linear dynamic and static non-linear elements.

3. Y. W. LEE and M. SCHETZEN 1965 International Journal of Control 2, 237-254. Measurement of the Wiener kernels of a non-linear system by cross-correlation.

4. E. ISOBE and S. SATO 1984 IEEE Transactions on Automatic Control, 29, 595-602. An integrodifferential formula on the Wiener kernel and its application to sandwich system identification.

5. R. E. WICKESBERG and C. D. GEISLER 1984 IEEE Transactions on Biomedical Engineering 31, 454-461. Artifacts in Wiener kernels estimated using Gaussian white noise.

6. A. B. GARDINER 1973 International Journal of Control, 18, 1029-1039. Identification of processes containing single-valued non-linearities.

7. R. V. WEBB 1974 Electronics Letters 10, 344-346. Identification of the Volterra kernels of a process containing single-valued non-linearities.

8. K. S. Shanmugam and M. T. JoNG 1975 IEEE Transactions on Aerospace and Electronic Systems 11, 1218-1225. Identification of non-linear systems in frequency domain. 
9. J. SANDOR and D. Williamson 1978 International Journal of Control 27, 853-878. Identification and analysis of non-linear systems by tensor techniques.

10. M. J. Korenberg 1973 Proceedings of the 16th Midwest Symposium on Control Theory pp. 2.1-2.9. Identification of biological cascades of linear and static non-linear systems.

11. M. J. KORENBERG 1973 Proceedings of the 10th Rocky Mountain Bioengineering Symposium pp. 47-52. Cross-correlation analysis of neural cascades.

12. S. A. BILlings and S. Y. FAKHOURI 1978 IEE Proceedings on Control and Science 125, 1051-1058. Theory of separable processes with applications to the identification of non-linear systems.

13. S. A. BILLINGS and S. Y. FAKHOURI 1980 International Journal of Systems Science 11, 261-279. Identification of non-linear systems using correlation analysis and pseudo-random inputs.

14. S. Y. FAKHOURI 1980 International Journal of Systems Science 11, 541-555. Identification of a class of non-linear systems with Gaussian non-white inputs.

15. G. A. PARKER and E. L. MOORE 1982 Automatica 18, 85-91. Practical non-linear system identification using a modified Volterra series approach.

16. E. DE BOER, J. G. CORNelis and H. R. DE JONGH 1982 Proceedings of the 6th IFAC Symposium on Identification and System Parameter Estimation, pp. 325-330. Signal transformation by non-linearity as the foundation for identification procedures.

17. M. J. KORENBERG 1985 Proceedings of the 7th IFAC Symposium on Identification and System Parameter Estimation, pp. 421-426. Identifying noisy cascades of linear and static non-linear systems.

18. B. COOPER and A. H. FALkNER 1975 IEE Proceedings on Control and Science 122, 753-755. Identification of simple non-linear systems.

19. S. YASUI 1979 IEEE Transactions on Automatic Control 24, 230-242. Stochastic functional Fourier series, Volterra series, and non-linear system analysis.

20. K. J. ÅsTROM 1980 Automatica 16,551-574. Maximum likelihood and prediction error methods.

21. L. LJUNG 1987 System Identification: Theory for the User. Englewood Cliffs, NJ: Prentice-Hall.

22. S. M. PANDIT and S. M. WU 1983 Time Series and System Analysis with Applications. New York: John Wiley. 